Nussenzweig showed that the protein was expressed in the tumours derived from $\operatorname{Trp} 53^{-/-} \mathrm{H} 2 \mathrm{AX^{+/- }}$ mice as well, and both groups went on to show that the protein level in $\mathrm{H}_{2} \mathrm{AX} \mathrm{X}^{+/-}$thymocytes was about half that in $H 2 A X^{+/+}$thymocytes. Both groups also found that genomic instability in $H 2 A X$ heterozygotes was intermediate between that in wildtype and null cells. Finally, Nussenzweig found that the phosphorylation of H2AX on serine residues 136 and 139 is crucial for its ability to prevent genomic instability.

So, these mouse models indicate that $\mathrm{H} 2 \mathrm{AX}$ suppresses genomic instability and tumorigenesis. Whether it has the same effect in humans remains to be established.

Emma Greenwood

\section{(6) References and links}

ORIGINAL RESEARCh PAPERS Bassing, C. H. et al. Histone H2AX: a dosage-dependent suppressor of oncogenic translocations and tumors. Cell 114, 359-370 (2003) | Celeste, A. et al. H2AX haploinsufficiency modifies genomic instability and tumor susceptibility. Cell 114, 371-383 (2003)

\section{WEB SITES}

Frederick Alt's lab:

http://www.hms.harvard.edu/dms/bbs/fac/alt.htm

Andre Nussenzweig's lab:

http://rex.nci.nih.gov/RESEARCH/basic/eib/nusenzwg.htm

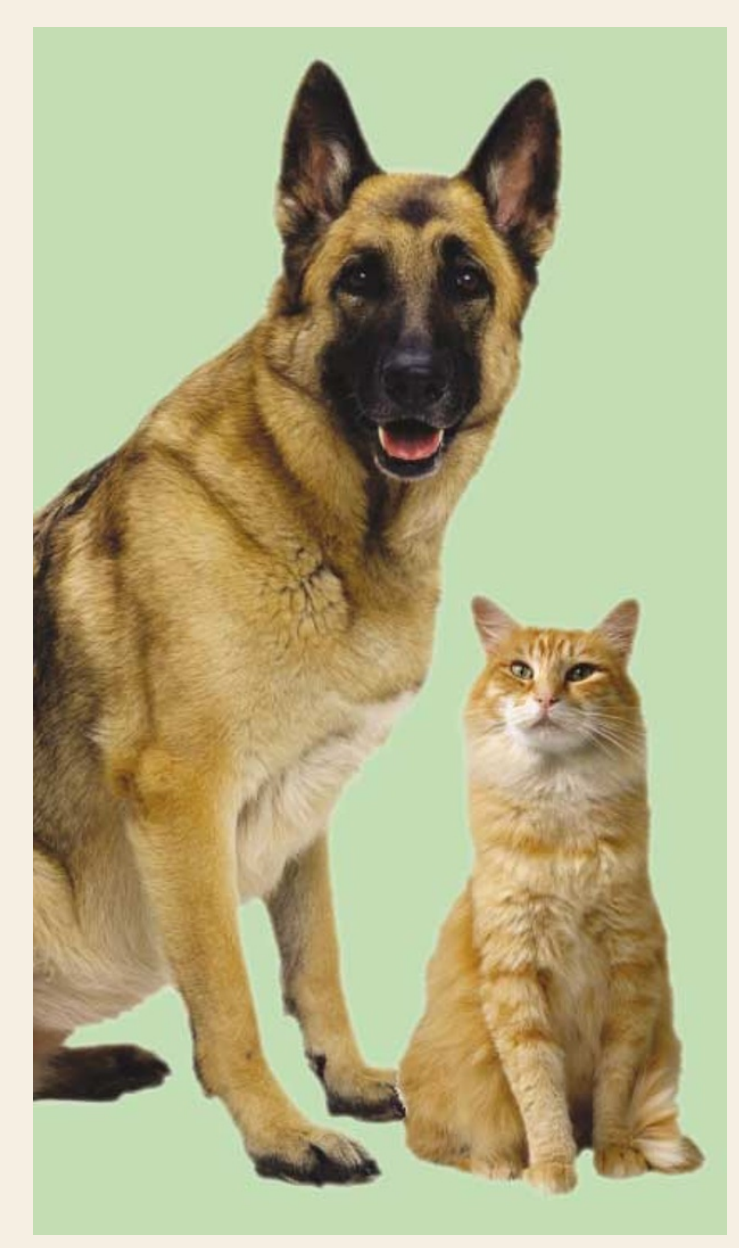

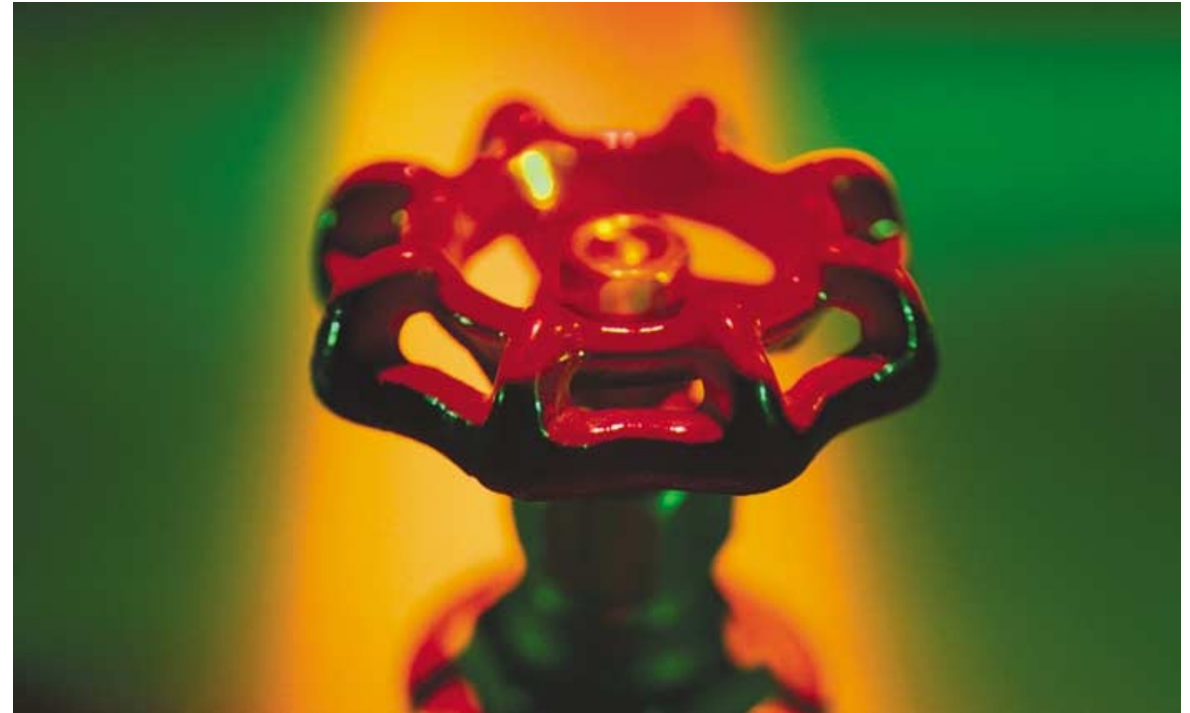

TUMORIGENESIS

\title{
Stopping the leaks
}

Compared with normal blood vessels, tumour vasculature is extremely leaky. This is thought to aid tumour progression, as plasma proteins can enter the surrounding tissue to provide a fertile environment for tumour growth. In the July issue of Cancer Cell, William Sessa and colleagues have now found that plugging the leaks in tumour blood vessels actually blocks tumour growth in mice.

The authors have previously shown that cavtratin - a chimeric peptide containing the scaffolding domain of caveolin-1 linked to a cellular internalization sequence - reduces vascular permeability in mouse models of inflammation. Caveolin-1 is the primary coat protein of caveolae and has been shown to regulate endothelial nitric-oxide synthase (eNOS), which controls vascular remodelling and angiogenesis and is activated by vascular endothelial growth factor (VEGF). They began by investigating the effects of cavtratin on VEGF-mediated vascular leakage by measuring Evans blue-dye extravasation - an indicator of albumin and plasma-protein leakage - after intradermal administration of VEGF. Mice pretreated with cavtratin showed a marked reduction in Evans blue-dye extravasation compared with control mice pretreated with a control peptide AP-CAV-X. So, as cavtratin reduces VEGF-mediated vascular permeability, does it have a similar effect on tumour blood-vessel permeability?

Mice with Lewis-lung-carcinoma (LLC) tumours were treated with cavtratin or AP-CAV-X, but only cavtratin inhibited tumour vascular leakage without affecting vascular permeability in the lungs. As the effects of cavtratin were tumour specific, they monitored tumour progression in the LLC model and found that daily administration of cavtratin significantly reduced tumour size. Cavtratin, but not AP-CAV-X, reduced tumour-derived
NOS activity and significantly reduced expression of platelet-endothelial-cell adhesion molecule-1 (PECAM-1) - a common marker for tumour endothelium - and the VEGF receptor FLT4 a marker for lymphatic vasculature. Histological examination identified large areas of necrosis in cavtratin-treated tumours, and cavtratin increased the number of apoptotic cells in non-necrotic regions of the tumour. So, cavtratin reduces eNOS activity, decreases tumour permeability and causes apoptosis.

Direct effects of cavtratin on angiogenesis, endothelial-cell proliferation and tumour-cell growth were ruled out and the role of eNOS in vascular leakage was investigated further. Comparison of LLC tumours in wild-type and $e \mathrm{NOS}^{-/-}$mice showed that tumours from $e^{N_{O}}{ }^{-1-}$ mice were less permeable to Evans blue-dye than tumours from wild-type animals. Administration of cavtratin to wild-type animals reduced tumour permeability to the same level seen in $e \mathrm{NOS}^{-/-}$tumours. In addition, $e \mathrm{NOS}^{-/-}$mice have reduced vascular permeability, tumour growth and sensitivity to cavtratin, compared with wild-type mice, providing further evidence that eNOS is the primary molecular target of cavtratin. As treatment of endothelial cells with cavtratin did not block VEGF-induced autophosphorylation of the VEGF receptor FLK1 or phosphorylation of c-SRC, genetic loss of eNOS is comparable to inhibition by cavtratin.

These results indicate that caveolin- 1 is a novel target for antitumour therapy, as it specifically affects tumour microvasculature.

Emma Croager

\section{(D) References and links}

ORIGINAL RESEARCH PAPER Gratton, J.-P. et al. Selective inhibition of tumor microvasculature permeability by cavtratin blocks tumor progression in mice. Cancer Cell 4, 31-39 (2003) WEB SITE

William Sessa's lab:

http://info.med.yale.edu/pharm/sessa/index.html 